\title{
Discrepancies Identified with the Use of Prescription Claims and Diagnostic Billing Data Following a Comprehensive Medication Review
}

\author{
Teresa E. Roane, PharmD, BCACP; Vinita Patel, PharmD; \\ Heather Hardin, PharmD, BCACP; and Martha Knoblich, PharmD
}

\begin{abstract}
BACKGROUND: The University of Florida College of Pharmacy's Medication Therapy Management Communication and Care Center (UF MTMCCC) provides medication therapy management (MTM) services to patients enrolled in a State of Florida Medicaid Waiver Program: Medicaid for the Aged and Disabled. To provide these services, UF MTMCCC was given access to patients' prescription claims data and diagnostic billing data in the form of ICD-9 codes. Prior to calling a patient, a precomprehensive medication review (CMR) work-up was performed to identify potential medicationrelated problems (MRPs) and/or health-related problems (HRPs). Based on information provided by the patient in relation to comorbidities, medications, and medical history during the interactive telephone conversation, problems were either confirmed or eliminated. All of the reported information was also assessed to identify any new MRPs or HRPs. Accordingly, telephonic MTM services have the potential to bridge the gap between pharmacy claims data and patient self-reported information, since the MTM services provided rely on the accuracy of both informational resources.
\end{abstract}

OBJECTIVE: To determine the degree of discrepancy in patient-reported information regarding chronic comorbidities and medications versus diagnostic billing data (ICD-9 codes for chronic comorbidities) and pharmacy claims data (medications) when providing MTM services during an interactive telephonic comprehensive medication review.

METHODS: A retrospective chart review ( $n=147$ patients) was performed for patients who received a telephonic CMR. Pharmacy claims data and diagnostic billing data, in conjunction with the pre-CMR work-up data, were used to identify discrepancies in information obtained from the patient during the CMR. During the chart review, identified MRPs or HRPs were categorized as "confirmed" (patient reported the problem exists and/or it was deduced from the presence/absence of a medication that the problem does exist); "eliminated" (patient reported the problem does not exist and/or it was deduced from the presence/absence of a medication that the problem does not exist); or "new" (a problem that was not identified during precall identification of problems, but following the CMR interaction, it was determined that a problem now exists). The study evaluated the discrepancies before and after a CMR telephonic interaction in the following categories: medications, chronic comorbidities, level 1 drug-drug interactions, level 2 drug-drug interactions, gaps in therapy, therapeutic duplications, lack of therapy, preferred drug list alternatives, combination products, and tobacco use. Percent discrepancy was calculated as the sum of new and eliminated data elements divided by the total number of data elements for each MRP or HRP.

RESULTS: The percent discrepancy observed was $42 \%$ for medications, $41 \%$ for chronic comorbidities, $77 \%$ for level 1 drug-drug interactions, $93 \%$ for level 2 drug-drug interactions, $35 \%$ for gaps in therapy, $87 \%$ for therapeutic duplications, $26 \%$ for lack of therapy, $36 \%$ for preferred drug list alternatives, $42 \%$ for combination products, and $54 \%$ discrepancy for report of tobacco use. 0verall, 4,441 data elements were identified as confirmed, eliminated, or new across the 147 CMRs. Among those data elements, $56 \%$ of the data was confirmed; $23 \%$ was eliminated; and $21 \%$ was discovered as new.

CONCLUSIONS: The study met its objective in determining the degree of discrepancies that existed when prescription claims data and ICD-9 billing data were used to identify MRPs and/or HRPs versus using patient-reported data. Data revealed that the presence of discrepancy is relatively large depending on the category, indicating a difficulty in accurately making recommendations with incomplete data or solely based on prescription claims and billing data. MTM services with patient interaction are vital in identifying information that allows for more appropriate decision making.

J Manag Care Pharm. 2014;20(2):165-73

Copyright $\odot$ 2014, Academy of Managed Care Pharmacy. All rights reserved.

\section{What is already known about this subject}

- The congruence of medication information from patient selfreporting and pharmacy claims data is variable and dependent on the time interval for claims data surveillance.

- Telephonic services have been used for providing general patient care and assessing the accuracy of pharmaceutical claims data.

- Electronic pharmacy records have been used for the assessment of medication compliance and adherence to disease state guideline recommendations.

- Drug use review using prescription database information and inferred diagnoses is widely used to trigger medication-related interventions.

- The combination of electronic medical record assessment with telephonic services has been employed for the provision of MTM services.

\section{What this study adds}

- Using the patient's electronic record and a telephone interview, data analyzed were the change in medications and perceived medication- and/or health-related problems between pre-CMR and post-telephonic CMR assessment.

- Results indicate that an assessment of the electronic medical record (prescription and billing claims) coupled with telephonic services leads to a more thorough medication review and assessment when providing MTM services. 


\section{Discrepancies Identified with the Use of Prescription Claims and Diagnostic Billing Data Following a Comprehensive Medication Review}

$\mathrm{M}$ edication therapy management (MTM) is "a distinct service or group of services that optimize therapeutic outcomes for individual patients." ${ }^{-3}$ MTM is further defined as patient-centered services that evaluate the patient's complete medication therapy regimen rather than focusing on an individual medication. ${ }^{2}$ The American Pharmacists Association and the National Association of Chain Drug Stores Foundation developed a model framework for implementing effective MTM services. The intent of this framework is to help improve collaboration among health care professionals, enhance communication between patients and the patients' health care team, and to optimize medication use for improved patient outcomes. ${ }^{2}$ Using the elements of the framework, the pharmacist, or other qualified health care professional, provides MTM services to patients to help enhance patients' knowledge of their medications and obtain the most benefit from those medications, as well as empower patients to assume a more active role in managing their medication therapy and their health conditions.

There are 5 core elements identified in the MTM service model framework. The first core element is the medication therapy review (MTR), also commonly referred to as a comprehensive medication review (CMR). ${ }^{2}$ The MTR/CMR is a systematic method of gathering patient-specific information using prescription claims data and information obtained during an interactive consultation with the patient or the patient's caregiver. The MTR/CMR also consists of gathering information on all of the medications the patient is currently taking that is not captured in the prescription claims data, including overthe-counter products, herbal therapies, homeopathic remedies, sample medications, and dietary supplements. All of the information gathered is then assessed to identify medication-related problems (MRPs) and/or health-related problems (HRPs), followed by the generation of a plan to resolve those problems that includes collaboration with the pharmacist, patient, caregiver, and/or the prescriber.

The second core element is the personalized medication record (PMR). ${ }^{2}$ The PMR is a comprehensive record, or medication list, containing all of the patient's prescription and nonprescription medications and is intended to be given to, and used by, the patient. The third core element is the medication action plan (MAP). ${ }^{2}$ The MAP is a document that is also intended for use by the patient and contains beneficial information for the patient to help in self-management of medications and conditions. The fourth element is intervention and/or referral. ${ }^{2}$ This element defines the pharmacist's role in providing consultative services and interventions to address medication-related problems with a referral to the appropriate health care provider when the pharmacist deems necessary. The final core element of the service model framework is the use of documentation with follow-up reviews and/or appointments. ${ }^{2}$ Documentation and follow-up are necessary components to maintain consis- tency throughout the process of providing MTM services, as well as to ensure the continuity of care for the patient. The method of providing MTM services using the core element framework involves using interactive encounters between the pharmacist and the patient and/or the patient's caregiver. The interactive encounter may be face-to-face, telephonic, or a combination of both methods.

The University of Florida College of Pharmacy established an MTM Communication and Care Center (UF MTMCCC) in March 2010. The center uses the fundamentals of the MTM service model framework to provide MTM services to patients. MTM services provided by UF MTMCCC used information obtained from prescription claims data and diagnostic billing data as well as patient self-reported data obtained during the CMR. The UF MTMCCC relies on patient self-reporting of information to confirm, eliminate, and identify new MRPs and/ or HRPs during the provision of MTM services.

The agreement between pharmacy data and self-reported data has been investigated in various settings. One study found that among chronic glucocorticoid users enrolled in a managed care program, agreement between self-report and osteoporosis care was high but was dependent on the time interval for pharmacy data review (Table 1). Specifically, the investigators reported an optimal interval of pharmacy data surveillance of 120-180 days to distinguish between current and past bisphosphonate users. ${ }^{4}$ Similarly, self-reported information was found to be more reliable than pharmacy claims data when the focus was on assessing patients' medications at a specific point in time. ${ }^{5}$ A patient's self-reported medical history is assumed to be accurate, and the validity of the reported medical information beyond medication lists has been investigated. In particular, an evaluation of the congruence of medical record information and self-reported history of preeclampsia found validity was only moderate. ${ }^{6}$ In addition, literature regarding the accuracy of selfreported history among autoimmune disease, schizophrenia, and chronic pain sufferers is also available (Table 1). ${ }^{7-9}$

Telephonic MTM services have the potential to bridge the gap between pharmacy claims data and patient self-reported information, as they rely on the accuracy of both informational resources. Telephonic interventions have also been employed to investigate the accuracy of available medication information. A 2004 assessment of the accuracy of computerized medication histories included patients aged at least 65 years who were receiving 5 medications or more. ${ }^{10}$ Only $5.3 \%$ of patients included in the assessment had complete agreement between the computer-generated medication list and the patient-reported medication history taken during the telephonic interview. ${ }^{10}$ Of all medications, $65 \%$ were prescription; $23 \%$ were over the counter (OTC); and 12\% were vitamins/ herbals. The average number of drug omissions was 3.1 per patient. Also, 25\% of the total number of medications reported by patients as actually being taken was found to be omitted 


\section{TABLE 1 Literature Regarding Patient Self-Reporting and Telephonic Services for General Care}

\begin{tabular}{|c|c|c|c|c|}
\hline Author, Year & Population & Sample Size & Method & Results \\
\hline $\begin{array}{l}\text { Curtis et al., } \\
2006^{4}\end{array}$ & $\begin{array}{l}\text { Chronic glucocorticoid users } \\
\text { from a National Managed Care } \\
\text { Organization database }\end{array}$ & 2,363 & $\begin{array}{l}\text { Compared self-reported current } \\
\text { use of alendronate, risedronate, } \\
\text { calcitonin, and raloxifene with dif- } \\
\text { ferent intervals of pharmacy data } \\
\text { to determine agreement. }\end{array}$ & $\begin{array}{l}\text { The } 6 \text {-month interval of pharmacy data failed to } \\
\text { capture }>25 \% \text { of self-reported current bisphospho- } \\
\text { nate users. The optimal interval for surveillance to } \\
\text { distinguish between current and past users was 120- } \\
180 \text { days. }\end{array}$ \\
\hline $\begin{array}{l}\text { Caskie et al., } \\
2006^{5}\end{array}$ & $\begin{array}{l}\text { Subset of individuals tested } \\
\text { during the seventh wave of the } \\
\text { Seattle Longitudinal Study }\end{array}$ & 1,430 & $\begin{array}{l}\text { Compared brown bag data collec- } \\
\text { tion information with pharmacy } \\
\text { database claims for the previous } 4 \\
\text { months. }\end{array}$ & $\begin{array}{l}\text { More than half the sample ( } 58 \% \text { ) had complete } \\
\text { agreement on all } 16 \text { of the chosen drug classes. } \\
\text { Chronic disease status was a significant predictor of } \\
\text { agreement between brown bag and pharmacy data } \\
\text { for all age groups. }\end{array}$ \\
\hline $\begin{array}{l}\text { Bourgeois } \\
\text { et al., } \\
2007^{7}\end{array}$ & $\begin{array}{l}\text { Patients and parents presenting } \\
\text { to an ED waiting room in a ter- } \\
\text { tiary care children's hospital }\end{array}$ & 936 & $\begin{array}{l}\text { Measured the sensitivity and spec- } \\
\text { ificity of } 3 \text { data sources for assign- } \\
\text { ing patients to disease categories. }\end{array}$ & $\begin{array}{l}\text { Disease category assignment based on patient- } \\
\text { reported information was significantly more sensitive } \\
\text { in correctly identifying as disease category than data } \\
\text { used by national disease surveillance systems. }\end{array}$ \\
\hline $\begin{array}{l}\text { Don and } \\
\text { Carragee, } \\
2008^{8}\end{array}$ & $\begin{array}{l}\text { Cohort of consecutive patients } \\
\text { with persistent axial pain after } \\
\text { an MVA from } 5 \text { spine specialists' } \\
\text { outpatient clinics }\end{array}$ & 335 & $\begin{array}{l}\text { Determined the validity of self- } \\
\text { reported history in patients with } \\
\text { pain in a retrospective, multiclinic } \\
\text { study. }\end{array}$ & $\begin{array}{l}\text { The self-reported rates of alcohol abuse, illicit drug } \\
\text { use, psychological diagnosis, and prior axial pain } \\
\text { were significantly lower than seen in the medical } \\
\text { records. }\end{array}$ \\
\hline $\begin{array}{l}\text { Brill et al., } \\
2007^{9}\end{array}$ & $\begin{array}{l}\text { Schizophrenic or schizoaffective } \\
\text { disorder males, healthy males }\end{array}$ & 131 & $\begin{array}{l}\text { Compared contemporaneous and } \\
\text { retrospective reports from a behav- } \\
\text { ioral functioning assessment. }\end{array}$ & $\begin{array}{l}\text { No overall significant differences found in accuracy } \\
\text { of reporting between persons with schizophrenia } \\
\text { and those without. }\end{array}$ \\
\hline $\begin{array}{l}\text { Kaboli et al., } \\
2004^{10}\end{array}$ & $\begin{array}{l}\text { Iowa VA Medical Center pri- } \\
\text { mary care patients aged } \geq 65 \\
\text { receiving at least } 5 \text { prescriptions }\end{array}$ & 493 & $\begin{array}{l}\text { Assessed accuracy of computerized } \\
\text { medication lists, allergies and ADR } \\
\text { records using telephonic inter- } \\
\text { views with patients. }\end{array}$ & $\begin{array}{l}\text { Patients were taking a mean of } 12.4 \text { medications: } \\
65 \% \text { prescription, } 23 \% \text { OTC, and } 12 \% \text { vitamin/herb- } \\
\text { als. Complete agreement between computer medica- } \\
\text { tion list and what patient was taking was found for } \\
\text { only } 5.3 \% \text { of patients. }\end{array}$ \\
\hline $\begin{array}{l}\text { Pit et al., } \\
2008^{11}\end{array}$ & $\begin{array}{l}\text { Community-dwelling, general } \\
\text { practice patients aged } \geq 65\end{array}$ & 566 & $\begin{array}{l}\text { Compared self-reported use of } \\
\text { medicines with pharmaceutical } \\
\text { claims data for different retrieval } \\
\text { periods using an agreement study. }\end{array}$ & $\begin{array}{l}\text { Kappa coefficients showed good to very good agree- } \\
\text { ment }(\geq 0.75) \text { with retrieval periods of } 30,60 \text {, and } 90 \\
\text { days for BZDs, low-risk NSAIDs, thiazide diuretics, } \\
\text { and most other drugs. }\end{array}$ \\
\hline
\end{tabular}

$A D R=$ adverse drug reactions; $B Z D=$ benzodiazepine; $E D=$ emergency department $H F=$ heart failure; $M V A=$ motor vehicle accident NSAID = nonsteroidal anti-inflammatory drug; OTC= over the counter; VA= Veterans Affairs.

from the electronic medical record. Likewise, an investigation of the agreement between medication lists, from telephonic self-reports versus claims data in Australia, reported that the agreement between the telephone self-reports and pharmacy claims data declined significantly as retrieval periods increased (7, 30, 60, and 90 days). ${ }^{11}$ The authors reported sensitivity and predictive values specific to classifications, with a marked decline in sensitivity being observed with increasing retrieval period for benzodiazepines $(88 \%, 80 \%$, and $74 \%$ for $30-, 60-$, and 90-day retrieval periods, respectively).

Assessing data received from pharmacy claims alone is not enough when providing MTM services. In today's world, patients are receiving medications from many different sources, including samples from their provider's office, through patient assistance programs, mailed in from other countries, or through their local pharmacy's free or low-cost medication programs. The interactive component of the CMR is vital in fully assessing the accuracy and completeness of a patient's medication history. The purpose of this study was to determine the degree of discrepancy between diagnostic billing codes (indicating chronic conditions/comorbidities), combined with pharmacy claims data (indicating active medications that were available prior to the patient interaction) and patient-reported health conditions and actual medication use data, obtained from the patient when MTM services were provided through an interactive telephonic consultation.

\section{Methods}

\section{Data Source}

A retrospective study was performed on 147 patients who received an interactive telephonic CMR consultation provided by the UF MTMCCC from June to August in 2011 as part of the patients' enrollment in the State of Florida Medicaid Waiver Program: Florida Medicaid for the Aged and Disabled (MEDS-AD) and MTM programs. This study received institutional review board approval from the University of Florida. Pharmacy claims data and diagnostic billing data were provided by the Agency for Health Care Administration. The pharmacy claims data provided a list of medications that were billed to and paid for by Florida Medicaid as a part of the patients' pharmacy benefits. The diagnostic billing data, in the form of the International Statistical Classification of Diseases and Related Health Problems, 9th Revision codes (ICD-9 codes), provided a list of conditions and services for the patient that 
were also billed to Florida Medicaid. These 2 sets of data were used together to identify a variety of plausible problems related to the patient's health care and were assessed prior to the telephone interactive consultation with the patient. The billed data were then compared with the patient-reported information obtained during the interactive CMR to identify discrepancies in the data.

\section{Patient Population}

The patients included in this study received MTM services provided by the UF MTMCCC and were enrolled in MEDS-AD. To be enrolled in the MEDS-AD program, patients were required to reside in the state of Florida, be Medicaid recipients assigned to the MEDS-AD Waiver program, and have an annual income at, or below, $88 \%$ of the federal poverty level with assets at, or below, $\$ 5,000$ for an individual (or $\$ 6,000$ for a couple). ${ }^{12}$ Also as part of the enrollment criteria, these patients could not be enrolled in a health maintenance organization plan. ${ }^{12}$

\section{Pharmacy Claims Data}

Prior to conducting a telephonic CMR, pharmacy claims data were used to generate a list of medications in the patient's chart. The precall medication list included any medications filled by the pharmacy and billed to Florida Medicaid for the patient from January 1, 2011, to the date of the actual CMR call. Medications excluded from this list included short-term antibiotic and antifungal regimens, prescription fills that did not overlap with the date of the CMR, or medications that were not considered to be used as long-term therapy for a chronic condition. Medications that were generally recognized to be dosed on a pro re nata (PRN), or "as needed" basis, were included in the list regardless of the days supply obtained or the date the prescription medication was filled. The rationale for including these PRN medications was the assumption that patients often keep PRN medications and have the potential to use them at any point throughout the year. Also, many PRN medications do not come with an end date alerting the patient when the treatment should be discontinued. Another reason for including the PRN medications was that the frequency of use for many of these medications may vary each month; therefore, the days supply may not correctly reflect how the medication was actually being used.

\section{Diagnostic Billing Data}

Diagnostic billing data in the form of ICD-9 codes were used to determine the chronic conditions each patient may have been diagnosed with, or received services for, prior to the interactive phone conversation. The ICD-9 codes that were provided for each patient dated back to January 1, 2009, and were current through the date of the patient's CMR. Because of repetition in the codes and conditions that would not normally be classified as chronic conditions, the billing data were reorganized so as to be applicable and meaningful for the purposes of this study. In order to reorganize successfully and consistently within the construct of this study, chronic conditions were only counted once as an issue was encountered to account for any repetition of the ICD-9 codes.

\section{Pre-CMR Identification of Problems}

In order to evaluate potential MRPs and/or HRPs, the medication list coupled with the information regarding chronic comorbidities was evaluated by the pharmacist prior to the CMR. Potential problems identified were to be addressed with the patient during the CMR call. Categorization of the MRPs and HRPs was based on information found in the core elements model framework of providing MTM services ${ }^{2}$ and included the following:

Drug-drug interactions (level 1 [severe] and level 2 [major]). A drug-drug interaction occurs when a medication affects the activity of another medication if the medications are administered simultaneously. For consistency within providing MTM services, Level 1 and Level 2 interactions were the focus of the drug-drug interaction report. Level 1 (severe) interactions and level 2 (major) interactions were classified using the Elsevier/Gold Standard drug information software database Clinical Pharmacology. ${ }^{13}$ The drug-drug interaction report was completed prior to the CMR and then analyzed to identify potential problems. The significance of the potential problems was assessed during the consultation with the patient. The interaction report was also updated and assessed, during or immediately after the call, as new medication information was obtained from the patient.

Therapeutic duplications. For the study purposes, a therapeutic duplication included any medication that was being filled as 2 different strengths of the same medication, or the filling of 2 different medications, in the same class of medications, which would not normally be considered a conventional therapy regimen (i.e., multiple angiotensin-converting enzyme inhibitors).

Gaps in therapy. A gap in therapy was defined as any medication that was missing for a specified chronic condition. The medication list generated by the pharmacy claims data was compared with the patient's chronic conditions to identify any potential gaps in therapy, and these gaps were then updated as information was obtained from the patient during the CMR interaction. The particular gaps that were considered during the study period were clearly defined and clinically accepted forms of therapy that have arisen from evidence-based medication, current therapy guidelines, and primary literature, and included applicable gaps in therapy as referenced in the START protocol (screening tool to alert doctors to the right treatment). ${ }^{14}$

Lack of therapy. A lack of therapy was defined as an indication in which there was not a corresponding medication being used for a particular indication or condition. Although there may be some overlap within the "gaps in therapy" section, the 
lack of therapy section takes into account any chronic condition, regardless of guideline-based therapy.

Preferred drug list alternatives. Florida Medicaid employs a Preferred Drug List (PDL) formulary system. The PDL is a list of medications that will be covered by Medicaid. Prescribers are encouraged to prescribe medications listed on the PDL when ordering medications for their patients. The patient's complete medication list was assessed for opportunities to use an equivalent medication as listed on the Florida Medicaid PDL.

Combination products and/or alternate dosing. A combination medication is a formulation of 2 or more active ingredients combined in a single dosage form. The patient's complete medication list was examined for the possibility of using combination products when applicable. The medication list was also assessed to identify whether the patient was taking multiple tablets of a particular strength of a medication when a higher dose tablet of that same medication was available. Both of these opportunities were used to help reduce the patient's daily pill burden and optimize current therapy.

Tobacco use. Using ICD-9 codes, patients with a history of smoking were identified prior to the CMR consultation. Actual tobacco use was then assessed during the CMR.

\section{Telephonic CMR Interaction}

The telephonic CMR interactions were completed by either licensed pharmacists or fourth-year student pharmacists on advanced clinical rotations under the direct supervision of a licensed pharmacist. During the CMR, each of the patient's preCMR medications was systematically assigned as "confirmed" (the patient stated they were still taking the medication) or "eliminated" (the patient stated they were no longer taking the medication). During the CMR, the patient was also asked the indication for each medication. The indication questioning allowed for either the confirmation or elimination of previously identified chronic conditions. Following the discussion of the medications identified prior to the call, the patient was also asked about any other medication that was being taken, including all OTC products, vitamins, dietary supplements, herbal medications, samples, and/or products obtained from any other source. These newly identified medications and their respective chronic comorbidities (if not previously identified) were then considered a "new" medication or "new" chronic comorbidity for the purposes of this analysis.

Following the review of the medications and chronic comorbidities, each of the potential problems identified prior to the call was assessed with the patient to confirm or eliminate the problem. This assessment was also the time for the identification of any new problems that occurred based on information provided by the patient (Table 2).

When related to tobacco use, "confirmed" was noted if the ICD-9 code provided evidence of tobacco use, and the patient reported the current use of tobacco products. The notation

\section{TABLE 2 Definitions of Confirmed,} Eliminated, or New Problems

\begin{tabular}{l|l}
\hline Problem Classification & \multicolumn{1}{c}{ Definition } \\
\hline Confirmed & $\begin{array}{l}\text { Patient reports the problem exists, and/or it is } \\
\text { deduced from the presence/absence of a medica- } \\
\text { tion that the problem does exist. }\end{array}$ \\
\hline Eliminated & $\begin{array}{l}\text { Patient reports the problem does not exist, and/or } \\
\text { it is deduced from the presence/absence of a } \\
\text { medication that the problem does not exist. }\end{array}$ \\
\hline A problem that was not identified during precall \\
identification of problems; however, following \\
the review of the medication list and/or chronic \\
conditions, it was determined that a problem \\
now exists.
\end{tabular}

"eliminated" was used if the ICD-9 code provided information of tobacco use, but the patient reported not currently using tobacco. Finally, the determination of "new" was used if no prior information about tobacco use was listed in the ICD-9 code history for the patient, and the patient reported during the CMR currently using tobacco products.

\section{Data Analysis}

A large variance was also observed with respect to medications, chronic comorbidities, and MRPs or HRPs that were listed as confirmed, eliminated or identified as new. A percent discrepancy for the variance in these items was then analyzed. The percent discrepancy was calculated as the sum of new and eliminated data elements divided by the total number of data elements for each category.

\section{Results}

For this study, 147 CMRs were completed out of 219 scheduled CMR appointments. Of patients asked to participate, 72 either refused the consultation or could not be reached after 3 attempts. Demographic data for the 147 patients that participated in the CMR are shown in Table 3. There was a wide variance in the amount of time spent on the phone with the patient during the CMR consultation. The minimum time for a telephonic encounter was 4 minutes and 10 seconds, and the maximum time for an encounter was 1 hour and 26 minutes. The average CMR consultation time was 33 minutes and 20 seconds.

There were a total of 4,441 data elements collected by the end of the study period. From these pieces of data, 2,469 were considered confirmed (56\%), while 1,024 (23\%) were considered eliminated. Of the total, 948 pieces of data (21\%) were identified as new MRPs or HRPs that were discovered during the CMR.

The percent discrepancy calculated for the categories identified was as follows: medications, $42 \%$; chronic comorbidities, 41\%; level 1 drug-drug interactions, $77 \%$; level 2 drug-drug 


\section{TABLE 3 Study Participant Demographics}

$\%$

\begin{tabular}{l|c|c}
\hline Age & & \\
\hline $10-19$ & 0.70 & 1 \\
\hline $20-29$ & 1.40 & 2 \\
\hline $30-39$ & 4.80 & 7 \\
\hline $40-49$ & 20.40 & 30 \\
\hline $50-59$ & 46.90 & 69 \\
\hline $60-64$ & 25.90 & 38 \\
\hline
\end{tabular}

\begin{tabular}{l|l|l}
\hline Sex & \multicolumn{2}{|c}{} \\
\hline Male & 44.00 & 64 \\
\hline Female & 56.00 & 83
\end{tabular}

Language

\begin{tabular}{l|r|r}
\hline English & 94.60 & 139 \\
\hline Spanish & 5.40 & 8 \\
\hline
\end{tabular}

interactions, 93\%; gaps in therapy, 35\%; therapeutic duplications, 87\%; lack of therapy, 26\%; preferred drug list alternatives, $36 \%$; combination products, $42 \%$; and tobacco use, $54 \%$. The percent discrepancy that was observed and calculated is shown in Table 4.

\section{Discussion}

Before discrepancies were actually analyzed, it was apparent that the data collected prior to a CMR encounter were vastly different from the data that were obtained from the patient during the interactive CMR consultation. Some of the newly identified discrepancies, such as drug-drug interactions and duplication of therapy, may have the potential to cause harm to the patient and may have been overlooked had the interactive consultation not been part of the actual medication review and assessment. Categorizing each data element in its proper data set provides better visualization of the impact of MTM services across the various categories captured in this study (Table 4).

\section{Medications and Chronic Comorbidities}

Data collected on medications alone had an overall discrepancy rate of $42 \%$, which shows that almost half of the information obtained from pharmacy claims data was not representative of the medications the patient was actually taking. Some of the reasons for the discrepancy in the medication category include patients receiving OTC products not covered by Medicaid (some OTC products are covered but not others), paying cash out of pocket for medications that were not covered by Medicaid, obtaining medications from their pharmacies via free medication campaigns, getting medications from out of the country, and patients receiving samples from their physicians.

Chronic comorbidities resulted in a $41 \%$ discrepancy, yielding the same impression that almost half of the data was not accurate. Reasons for this discrepancy may include patients being recently diagnosed with a condition not represented in the current ICD-9 codes, receiving testing for a condition that was later ruled out but still appeared as a current condition, or patient indicating never having been diagnosed with the condition listed. Consequently, these 2 data sets in turn had a downstream effect on the other 8 data sets, since medications and chronic comorbidities help indicate treatment options, interactions, and alternative medications and/or combinations. In addition, since medication and chronic comorbidities data sets are to help guide resolution of MRPs or HRPs, we did not anticipate that these data sets would show this degree of discrepancy.

\section{Drug-Drug Interactions}

Problems related to drug-drug interactions had an overwhelming number of eliminated data elements, which further highlights the benefit of providing interactive MTM services. Though there are many opportunities for medications to produce interactions when the medications are administered simultaneously, this study shows that the actual occurrence of a clinically significant interaction was much lower than anticipated. For level 1 drug-drug interactions, 23\% were confirmed, while $73 \%$ were actually eliminated. For level 2 drug-drug interactions, $7 \%$ were confirmed, while $77 \%$ were eliminated. By speaking with the patient and addressing interaction concerns, we discovered that about three fourths of suspected interactions were negligible or not considered clinically significant, meaning the patient was not reporting any of the symptoms related to the interaction, the interaction may have already been addressed by the patient's physician, or the patient was being followed more often by the physician through additional appointments or closer monitoring of laboratory values.

\section{Gaps in Therapy and Lack of Therapy}

Both the gaps in therapy and lack of therapy categories had different findings. Upon evaluating the data, results showed confirmed data at $65 \%$ and $74 \%$; eliminated data at $18 \%$ and $19 \%$; and new data at $16 \%$ and $7 \%$, respectively. A high confirmatory rate indicates that the medication for a condition or indication that appeared to be missing was truly missing from the therapy regimen. By identifying the patients that truly lack therapy or actually have a gap in therapy, this information can be relayed to the patient's primary health care providers so that the patients may benefit from the appropriate therapy that was previously lacking. Also, $16 \%$ of the gaps in therapy were identified after the consultation with the patient, which further highlights the validity of the idea that the review of claims data alone does not capture all the necessary information and supports the value of comprehensive interactive MTM services. These data also indicate that patients are going unrecognized 


\section{TABLE 4 Category Level Data for Study Parameters ${ }^{a}$}

\begin{tabular}{|c|c|c|c|c|c|c|c|c|c|c|c|}
\hline & \begin{tabular}{|rr} 
Medications \\
n
\end{tabular} & $\begin{array}{c}\text { Chronic } \\
\text { Comorbidities } \\
\mathbf{n} \quad(\%)\end{array}$ & \begin{tabular}{|c|} 
Level l \\
Drug-Drug \\
Interactions \\
$\mathrm{n} \quad(\%)$
\end{tabular} & \begin{tabular}{|c} 
Level 2 \\
Drug-Drug \\
Interactions \\
$\mathrm{n} \quad(\%)$
\end{tabular} & $\begin{array}{l}\text { Gaps in } \\
\text { Therapy } \\
\text { n (\%) }\end{array}$ & $\begin{array}{c}\text { Therapy } \\
\text { Duplications } \\
\text { n } \quad(\%)\end{array}$ & $\begin{array}{l}\text { Lack of } \\
\text { Therapy } \\
\text { n (\%) }\end{array}$ & \begin{tabular}{|c|} 
PDL \\
Alternatives \\
n $\quad(\%)$
\end{tabular} & $\begin{array}{c}\text { Combination } \\
\text { Products } \\
\text { n (\%) }\end{array}$ & $\begin{array}{cc}\text { Tobacco Use } \\
\text { n }\end{array}$ & $\begin{array}{l}\text { Total } \\
\text { n (\%) }\end{array}$ \\
\hline Confirmed & $1,141 \quad(58)$ & $676 \quad(59)$ & $5 \quad(23)$ & $20 \quad(7)$ & $96 \quad(65)$ & (13) & $457 \quad(74)$ & (64) & (58) & (46) & 2,469 \\
\hline Eliminated & $441 \quad(22)$ & $41 \quad(4)$ & (73) & $220 \quad(77)$ & (18) & $(85)$ & (19) & $2 \quad(14)$ & (42) & (19) & 1,024 \\
\hline New & $380 \quad$ (19) & $422 \quad(37)$ & (5) & $45 \quad(16)$ & (16) & $2 \quad(1)$ & 43 & (21) & (0) & (35) & \begin{tabular}{|ll}
$948 \quad(21)$ \\
\end{tabular} \\
\hline Total & 1,962 & 1,139 & 22 & 285 & 147 & 164 & 617 & 14 & 12 & 79 & 4,441 \\
\hline Discrepancy & 42 & 41 & 77 & 93 & 35 & 87 & 26 & 36 & 42 & 54 & \\
\hline
\end{tabular}

in the health care system and may be lacking clearly defined and clinically accepted forms of therapy, proven and accepted in both the primary literature and consensus guidelines created using evidence-based medication.

\section{Therapeutic Duplications}

The therapeutic duplication category had mostly eliminated data at a rate of $85 \%$. This high rate may have occurred from including all medications identified prior to the call versus making a clinical decision to not include medications that appeared to have been switched to a similar alternative. Though it may have been somewhat apparent based on the pharmacy claims data as to when one medication was switched to another, a concern always exists that the patient may continue to take both drugs simultaneously, which could potentially cause harm to the patient. This concern further promotes the use and value of providing MTM services, since there is potential for miscommunication and misunderstanding between the patient, the pharmacy, and the prescriber when the patient continues the use of medications that should have been discontinued.

\section{Preferred Drug List Alternatives and Combination Products}

While the category of PDL alternatives showed mostly confirmed data at a rate of $64 \%$, there was still a relatively large amount of eliminated and new data elements at $14 \%$ and $21 \%$, respectively. MTM services were beneficial in identifying opportunities for PDL alternatives and offer an opportunity for patients to accept to these alternatives. Similarly, the category of combination products had a high rate of confirmed data at $58 \%$ and also a high rate of eliminated data at $42 \%$.

Similar to the PDL alternatives, a high confirmed data rate for combination products represents the potential for improved adherence to medications because of a reduction in the patients' daily pill burden. These patients also agreed that a combination alternative would be an acceptable change in their daily medication regimens.

\section{Tobacco Use}

The amount of tobacco users that were confirmed based on the interactive consultation was at a rate of $46 \%$, while the amount eliminated was $19 \%$. Most importantly, this study showed that out of the 147 patients that received MTM services, at least 35\% of these patients were discovered to be new or current tobacco users that were not identified as such previously. The identification of new tobacco users created an excellent opportunity when providing MTM services. The interaction established a new group of patients that required smoking cessation counseling who may not have received the counseling previously, or may have never been approached with the questions during previous medical visits, since billing codes were not identifying these patients as smokers. MTM services presents the perfect opportunity to identify such patients while offering smoking cessation counseling during the interactive CMR consultation and then sending information to the patient regarding resources and tips to help the patient quit when the patient becomes ready. Also, with the incorporation of the core elements, including follow-up, these patients can be continuously monitored for successes, or relapses, in their efforts towards smoking cessation.

\section{Limitations}

While conducting this research study, a number of limitations were identified. First, the inclusion of PRN medications could have potentially caused the data to be skewed or presented more discrepancy within the data than necessary. For example, if the PRN medication was captured on the medication list prior to the call but the medication was indeed properly discontinued by the patient, it would have been added to the precall list of medications then later eliminated after the telephonic interaction, thereby possibly inflating the discrepancy percentages. Future analyses may want to set stricter guidelines on the inclusion or exclusion of PRN medications, or possibly create a separate category for PRN medications being used so that PRN medications may be assessed accordingly. 
Another limitation identified during the study was lack of a means to evaluate comorbidities that may have occurred greater than 2 years prior to the date of the consultation. Information may have been missing if that information was presented in the years prior to these 2 years. We anticipated that chronic comorbidities, such as diabetes or hypertension, would appear in the ICD-9 code billing data, since in accordance with standards of clinical care, these conditions should be re-evaluated on follow-up visits annually. However, health events such as a myocardial infarction or stroke that occurred prior to January 1, 2009, may not have been captured in more recent ICD-9 codes when reviewed prior to the CMR interaction. In retrospect, if each CMR interaction was designed to ask every patient about common comorbidities, other than those identified by ICD-9 codes or personally reported by the patient, there may have been a more complete picture of the patient and therefore a more thorough evaluation would have resulted.

The telephonic CMR interactions were conducted by either a pharmacist or a student pharmacist under the direct supervision of a pharmacist. This difference may also be considered a limitation as there may have been inconsistencies between the patient interactions performed by the pharmacist and the patient interaction performed by the student pharmacist under the supervision of a pharmacist. However, the majority of the CMR interactions were provided by the pharmacist, and the interview was structured and focused for both the pharmacist and student pharmacist, as previously described; therefore, inconsistencies or variances, if any, should be minimal. Additionally, the telephonic CMRs were conducted both for English-speaking and Spanish-speaking patients. The use of translators was necessary to conduct the telephonic CMR for this subset of patients; however, the translation was performed by a Spanish-speaking pharmacist in conjunction with the UF MTMCCC pharmacist or student pharmacist. Again, there is a concern regarding the consistency of information relayed back and forth during the consultation as well as how thorough the CMR is completed in such a setting with a language barrier and use of a pharmacist translator. Another concern during the call, and a limitation to the study, could be the occurrence of response bias, or the truthfulness or accurateness of the patient's answers during the interaction. The patient intentionally answering the question incorrectly to please, or not disappoint, the pharmacist may occur and would affect the outcome of the data being evaluated.

Finally, the findings of our study may not be generalizable to other populations. The patients eligible to receive the MTM services were selected from the MEDS-AD Waiver Program. These patients were from a lower socioeconomic status in society. As the demographic data represent, all patients included in the study were also less than 65 years of age. Consequently this population may not be representative of the traditional MTM population that would receive MTM services as a part of their Medicare Part D plan benefits. Also, there were no inclusion criteria for the patients in the study, other than the requirements of having active Medicaid services and being in the MEDS-AD Waiver Program, to participate in the program and receive UF MTMCCC services. Traditional MTM programs, as provided by Medicare Part D health plans, have inclusion criteria defined as a specific number of chronic medications with a certain number of chronic health conditions and a specified annual amount of drug spending for their Part D medications. Therefore, the data found in this study may not be generalizable to the traditional MTM population receiving MTM services from Medicare Part D providers and may be more generalizable to other Medicaid MTM programs.

\section{Conclusions}

MTM is defined as "a distinct service, or group of services, that optimize therapeutic outcomes for individual patients."1-3 Since it is a relatively new concept that is still evolving, we felt it was important to examine how effective the current methods for providing MTM services have been, as well as considering the benefit of the interactive patient consultation. This assessment has proven slightly challenging in the past and has given rise to many diverse programs and ways of carrying out MTM services. ${ }^{3,15}$ The UF College of Pharmacy established an MTM program that closely follows the definition and framework of the core elements for providing MTM services, while conducting telephonic interactive consultations with patients. This study set out to determine the degree of discrepancy between diagnostic billing codes (chronic conditions/comorbidities) and pharmacy claims data (medications) that was available prior to the patient interaction, as compared with patientreported health conditions and medication use obtained from the patient when providing MTM services through telephonic interactive consultations.

Whether considering each individual data set or looking at the picture as a whole, the objective of this study was clearly met. Our findings determined that there is a definite degree of discrepancy when comparing diagnostic conditions (chronic conditions/comorbidities) and pharmacy claims data (medications) with patient-reported data when providing MTM services through telephonic interactive consultations. With the discrepancy being so large, the value of MTM telephonic interactions can be realized. Therefore, it may be impossible to make an accurate recommendation when having incomplete data or data that contain such discrepancies. Through interactive consultations with patients and evaluation of the available data provided prior to, and as a result of, patient-reported information, health care providers can make more appropriate recommendations with the goal of improving patient outcomes. 


\section{Authors}

TERESA E. ROANE, PharmD, BCACP, is Clinical Assistant Professor, Department of Pharmacotherapy and Translational Research; VINITA PATEL, PharmD, is MTM Clinical Pharmacist; and HEATHER HARDIN, PharmD, BCACP, is Clinical Assistant Professor, MTM Communication and Care Center, University of Florida College of Pharmacy, Gainesville, Florida. MARTHA KNOBLICH, PharmD, is Pharmacy Resident, BayCare Health System, Tampa, Florida.

AUTHOR CORRESPONDENCE: Teresa E. Roane, PharmD, BCACP, Clinical Assistant Professor, University of Florida College of Pharmacy, Dept. of Pharmacotherapy and Translational Research, MTM Communication and Care Center, 2046 N.E. Waldo Rd., Eastside Campus Office Bldg., Ste. 2250, Gainesville, FL 32609. Tel.: 352.273.9692; Cell: 352.214.8382;

Fax: 352.273.9658; E-mail: troane@cop.ufl.edu.

\section{DISCLOSURES}

The University of Florida (UF) MTM Communication and Care Center receives grant funding from the Agency for Health Care Administration. The authors declare no potential conflicts of interest with respect to the research, authorship, and/or publication of this article.

Study concept and design were from Patel and Roane, and data collection was done by Patel and Knoblich. All authors were involved in data interpretation and writing the manuscript.

\section{ACKNOWLEDGMENTS}

The authors acknowledge the contribution of Shelley Stevens, PharmD, in the preparation of this manuscript.

\section{REFERENCES}

1. Bluml BM. Definition of medication therapy management: development of profession wide consensus. J Am Pharm Assoc (2003). 2005;45(5):566-72.
2. American Pharmacists Association; National Association of Chain Drug Stores Foundation. Medication therapy management in pharmacy practice: core elements of an MTM service model (version 2.0). J Am Pharm Assoc (2003). 2008;48(3):341-53. Available at: http://japha.org/article. aspx?articleid=1043461. Accessed September 10, 2013.

3. Pellegrino AN, Martin MT, Tilton JJ, Touchette DR. Medication therapy management services: definitions and outcomes. Drugs. 2009;69(4):393-406.

4. Curtis JR, Westfall AO, Allison J, Freeman A, Kovac SH, Saag KG. Agreement and validity of pharmacy data versus self-report for use of osteoporosis medications among glucocorticoid users. Pharmacoepidemiol Drug Saf. 2006;15(10):710-18.

5. Caskie GI, Willis SL, Warner Schaie K, Zanjani FA. Congruence of medication information from a brown bag data collection and pharmacy records: findings from the Seattle longitudinal study. Exp Aging Res. 2006;32(1):79-103.

6. Coolman M, de Groot CJ, Jaddoe VW, Hofman A, Raat H, Steegers EA Medical record validation of maternally reported history of preeclampsia. J Clin Epidemiol. 2010;63(8):932-37.

7. Bourgeois FT, Porter SC, Valim C, Jackson T, Cook EF, Mandl KD. The value of patient self-report for disease surveillance. J Am Med Inform Assoc. 2007;14(6):765-71.

8. Don AS, Carragee EJ. Is the self-reported history accurate in patients with persistent axial pain after a motor vehicle accident? Spine J. 2009;9(1):4-12.

9. Brill N, Reichenberg A, Rabinowitz J, et al. Accuracy of self reported premorbid functioning in schizophrenia. Schizophr Res. 2007;97(1-3):103-08.

10. Kaboli PJ, McClimon BJ, Hoth AB, Barnett MJ. Assessing the accuracy of computerized medication histories. Am J Manag Care. 2004;10(11 Pt 2):872-77.

11. Pit SW, Byles JE, Cockburn J. Accuracy of telephone self-report of drug use in older people and agreement with pharmaceutical claims data. Drugs Aging. 2008;25(1):71-80.

12. Florida Medicaid. Medicaid prescribed drug program: spending control initiatives, for quarters ended September 30, 2010 and December 31, 2010. Available at: http://ahca.myflorida.com/Medicaid/Prescribed_Drug/pdf/ SFY_1011_Q1Q2_FINAL.pdf. Accessed September 10, 2013.

13. Clinical Pharmacology [online database]. Elsevier/Gold Standard. 2012 Available at: http://www.goldstandard.com/product/drug-reference-patienteducation/clinical-pharmacology/. Accessed November 18, 2013.

14. Barry PJ, Gallagher P, Ryan C, O'Mahony D. START (screening tool to alert doctors to the right treatment) —an evidence-based screening tool to detect prescribing omissions in elderly patients. Age Ageing. 2007;36(6):632-38.

15. Shoemaker SJ, Hassol A. Understanding the landscape of MTM programs for Medicare Part D: results from a study for the Centers for Medicare and Medicaid Services. J Am Pharm Assoc (2003). 2011;51(4):520-26. 